\title{
Retraction Note: Outcomes of length-stable fixation of femoral neck fractures
}

Sreevathsa Boraiah • Omesh Paul • Michael J. Gardner •

Robert J. Parker · Joseph U. Barker •

David Helfet $\cdot$ Dean Lorich

Published online: 13 March 2012

(C) Springer-Verlag 2012

Retraction Note: Arch Orthop Trauma Surg (2010)

130:1523-1531

DOI 10.1007/s00402-010-1103-6

This article has been retracted due to plagiarism.

The online version of the original article can be found under doi: 10.1007/s00402-010-1103-6.

S. Boraiah $(\bowtie) \cdot$ O. Paul · M. J. Gardner .

R. J. Parker · J. U. Barker · D. Helfet · D. Lorich

Orthopaedic Trauma Service, 520 East 70th Street,

New York, NY 10021, USA

e-mail: bsreevathsa@ rediffmail.com; vathsaboraiah@gmail.com 